\title{
More light \\ from organic light-emitting diodes
}

— Wolfgang Brütting, Jörg Frischeisen, Bert J. Scholz, and Tobias D. Schmidt - DOI: 10.1051/epn/2011402

— Institute of Physics, University of Augsburg, 86135 Augsburg, Germany - Email: wolfgang.bruetting@physik.uni-augsburg.de

Organic light-emitting diodes (OLEDs) are efficient large-area light sources facing their market entry. Still, the development of stable and more efficient blue emitters and the enhancement of light outcoupling remain challenges for further device improvements. Here, we review the working principles of OLEDs and highlight ongoing efforts to improve their efficiency.

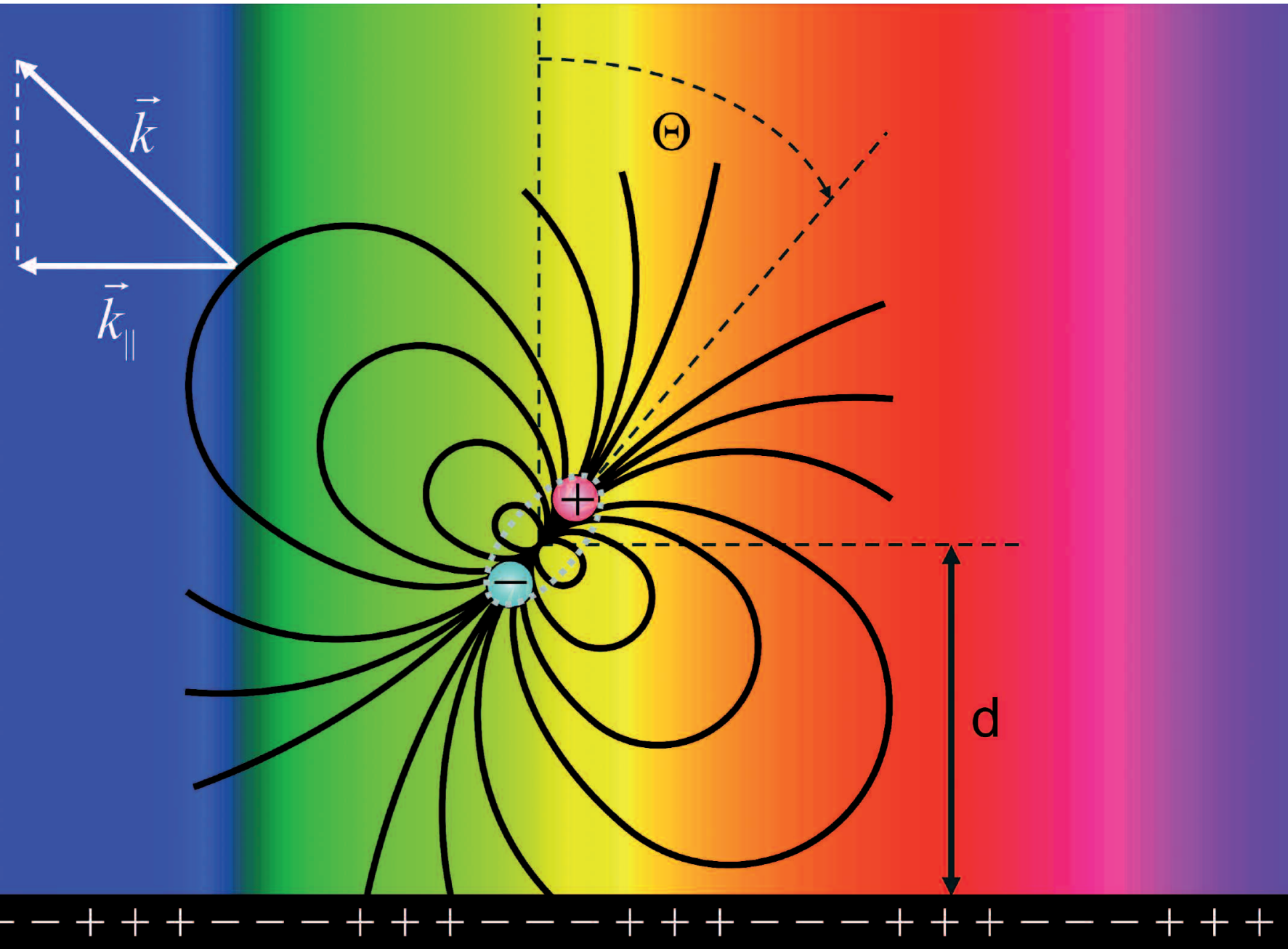


$\mathrm{T}$

he availability of artificial light has been a seminal cultural development of mankind. After open fires for thousands of years, the introduction of electricity together with the invention of the light bulb in the second half of the $19^{\text {th }}$ century has revolutionized our daily life. Having dominated the lighting market for more than 100 years, however, the light bulb faces its decline due to the need for technologies that convert electricity more efficiently into visible light. Besides fluorescent lamps, which are already well established in the market, the availability and progress in white light-emitting diodes, both inorganic and organic, has led to a new lighting technology called solid-state lighting over the last decade. Its working principle, namely the radiative recombination of injected electron-hole pairs in a solid, a process termed electroluminescence, is fundamentally different from existing techniques and holds the promise for highly efficient, long-lived and environmentally friendly light sources.

In contrast to their inorganic counterparts, organic light-emitting diodes (OLEDs) are flat and thin largearea light sources that could rather lead to complementary luminaires than competitors. Historically speaking, electroluminescence in organic molecular crystals dates back to the early 1960s. However, the important step towards applicable devices was the demonstration of thin-film organic light-emitting diodes by researchers at Eastman Kodak in 1987 using vacuum-deposited molecular materials and by a group at Cambridge University in 1990 making use of a solution-processed conjugated polymer [1]. Inspired by these publications, intense research and development throughout the 1990s has lead to the introduction of first commercial products based on OLED displays. Since 2000, however, the focus in many laboratories shifted towards OLEDs for lighting applications, i.e., to white OLEDs. After steady improvements in efficiency and lifetime over the years, the commercialization of OLEDs for general lighting has just recently started in 2010 [2]. (For an overview of various technical aspects of OLED lighting and the current state of the art see, e.g., Ref. [3]).

\section{White OLEDs}

The first report on white OLEDs dates back to 1994, when a Japanese group combined red, green and blue (RGB) laser dyes in a common matrix and achieved light emission over a broad spectral range [4]. Despite its simplicity in preparation, achieving white light emission with good and stable colour quality in this way is not that straightforward, because charge recombination and energy transfer processes between the different dyes need to be well controlled. Thus it is nowadays more common to employ distinct emission layers for

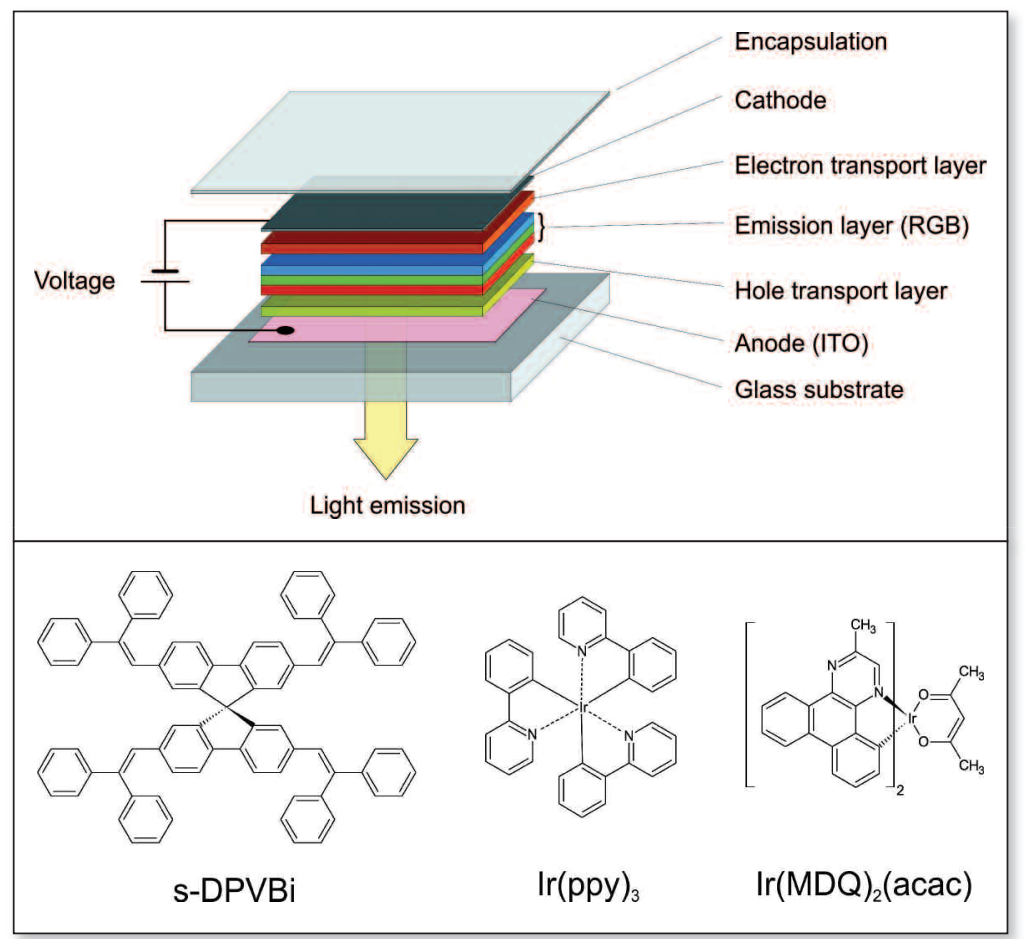

$\triangle$ FIG. 1: Typical layer stack of a small molecule OLED with a hole injecting and transporting unit deposited onto an optically transparent indium tin-oxide anode sitting on a glass substrate, followed by one or more emission layers (RGB), an electron delivering unit on top of it and finally an opaque metal electrode at the top. The whole layer stack is protected against ambient environment by an encapsulation which is typically a second glass plate combined with a desiccant. At the bottom some prototype OLED emitter materials are shown. In this case, the material for blue (s-DPVBi) is a fluorescent emitter, while green $\left(\operatorname{Ir}(\mathrm{ppy})_{3}\right)$ and $\operatorname{red}\left(\operatorname{Ir}(\mathrm{MDQ})_{2}(\mathrm{acac})\right)$ are phosphorescent emitters.

RGB that can either be directly stacked on top of each other in one OLED (see Fig. 1) or in three separated OLEDs - one for each colour - that are in turn vertically stacked by optically transparent electrical interconnecting units.

The development of OLEDs will continue to rely on the availability of tailor-made functional organic materials that can be applied to well-controlled thin films in the 10 to 100 nanometres thickness range. Thereby the requirements to the materials are manifold: starting from processibility and film formation, via electrical transport to optical properties. The key factor is obviously the availability of efficient and stable light emitters in the full visible spectral range. In this respect one has to distinguish bet-

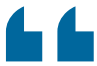
ween fluorescent and phosphorescent materials (see box). A seminal step was the introduction and further development of emitters based on heavy-metal centred metal-organic complexes, as shown in Fig. 1, where strong spin-orbit coupling mixes singlet and triplet states much more than in pure hydrocarbons, so that phosphorescence becomes an allowed transition [5]. 
In the meantime, impressive efficiency data have been published for OLEDs based on these materials [6], however, the bottleneck is still the limited availability and stability of deep-blue phosphorescent emitters.

\section{Optical losses and light outcoupling}

With the above said, the internal quantum efficiency of OLEDs can be brought up towards the theoretical limit of $100 \%$, if charge carrier injection and recombination are well balanced, if phosphorescent emitters are used and if non-radiative exciton quenching processes are suppressed (see also box). Nevertheless, only a fraction of the light will in the end be able to leave the device to the outside world. The reason is that light is generated in a region of the OLED stack with higher refractive index than the glass substrate and, obviously, ambient air. Thus, an excited molecule can couple to different optical modes in such a thin film structure (see Fig. 2). Viewed from the emitter position the light-escape cone has an opening angle of some $30^{\circ}$ with respect to the surface normal and the energy it contains is typically less than $20 \%$ of the total energy. This is followed by the

BOX 1: Working principle of OLEDs and their efficiency

The external quantum efficiency $\eta_{\mathrm{EQE}}$ of an OLED, i.e., the number of emitted photons per injected carrier pair, is given as the product of four different factors, each standing for a specific physical process in the device [14]:

$$
\eta_{\mathrm{EQE}}=\gamma \cdot \eta_{\mathrm{S} / \mathrm{T}} \cdot q_{\mathrm{eff}} \cdot \eta_{\text {out }}
$$

Therein $\gamma$ is the charge-carrier balance factor, describing whether or not equal amounts of electrons and holes are injected and what fraction of them recombines to form an exciton, as indicated in Fig. The second factor $\eta_{\mathrm{S} / \mathrm{T}}$ gives the fraction of excitons that is allowed to decay radiatively by spin statistics. As both, electrons and holes, carry spin $1 \frac{1}{2}$ there are three possibilities to form a triplet exciton with total spin $S=1$ and just

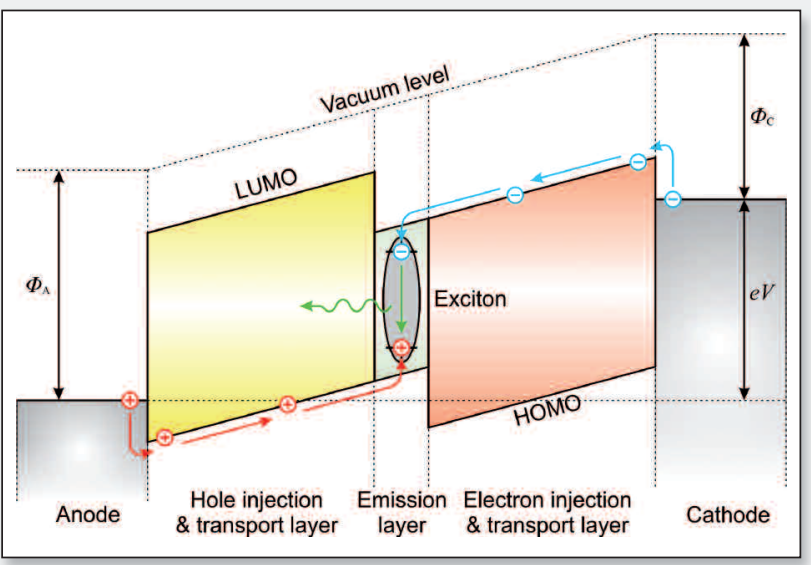

one for the formation of a singlet exciton with $\mathrm{S}=0$. For fluorescent emitters only singlets are allowed to decay radiatively making this factor 0.25 in this case, while for phosphorescent emitters, where singlets as well as triplets can emit light, it is 1 . The third factor $q_{\text {eff }}$ indicates how many of the spin-allowed excitons actually do decay by emitting a photon (instead of dissipating the excitation energy non-radiatively to their environment). Finally, the last factor $\eta_{\text {out }}$ determines which fraction of the generated photons are in the end able to leave the device to the outside world. Hence the external quantum efficiency can be split into an internal quantum efficiency $\left(\eta_{\text {IQE }}\right)$ times the outcoupling factor $\left(\eta_{\text {out }}\right)$.
To quantify the amount of light reaching the observer, one has to consider the sensitivity of the human eye. The luminous flux $\Phi_{L}$ (measured in Lumen $(\mathrm{Im})$ ) is obtained by multiplying the spectral flux of radiation $\Phi_{R}$ (measured in watt per nanometre $(\mathrm{W} / \mathrm{nm})$ ) with the response curve of the eye $(V(\lambda))$ and integrating over the visible spectral range (see Fig.):

$$
\Phi_{L}=K_{m} \cdot \int_{380 \mathrm{~nm}}^{780 \mathrm{~nm}} V(\lambda) \cdot \Phi_{R}(\lambda) d \lambda
$$

The photonic constant $K_{m}=683 \mathrm{~lm} / \mathrm{W}$ determines the maximum luminous efficacy obtained for a monochromatic green emitter at a wavelength of $555 \mathrm{~nm}$.

Given the electrical drive conditions (voltage $V$ and current $I$ ) and the luminous flux $\Phi_{L}$ it is possible to calculate the luminous efficacy $\eta_{L}$ according to:

$$
\eta_{L}=\frac{\Phi_{L}}{V \cdot I}
$$

4 Schematic energy level diagram showing the injection of electrons and holes from two electrodes with suitable work function $\left(\Phi_{\text {A/C }}\right)$ under an applied bias voltage (V). Charge carriers are transported either in the highest occupied molecular orbital (HOMO) of the hole transport layer or in the lowest unoccupied molecular orbital (LUMO) of the electron transport layer, respectively, before they recombine in the emission layer.

V Emission spectrum of a white OLED together with the sensitivity curve of the human eye and the spectrum of a light bulb at a radiation temperature of $3000 \mathrm{~K}$.

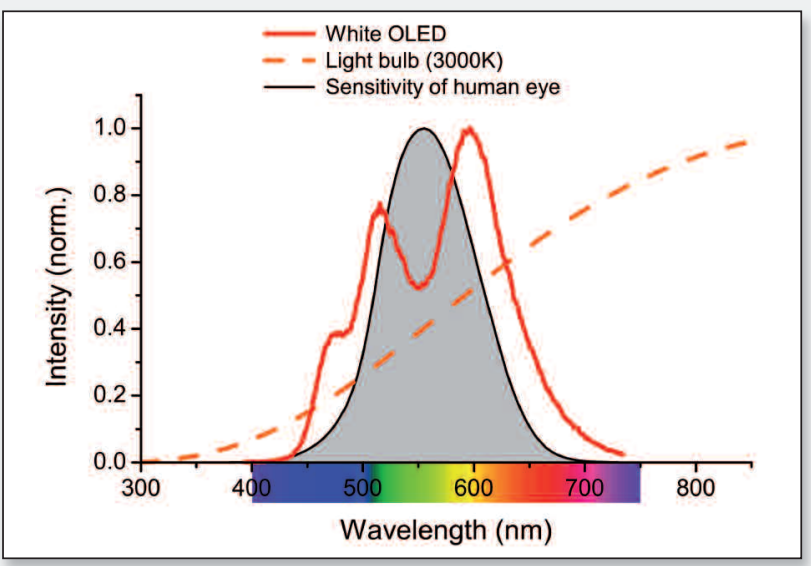


contribution of substrate modes, where total internal reflection at the glass-air interface is the limiting process. This contribution is comparable in energy also at around $20 \%$. For higher emission angles the light can not even reach the glass substrate, but is wave-guided in the organic layers (including the transparent indiumtin oxide electrode) and in the end lost by residual absorption or edge emission. Finally, the emitter can couple to the evanescent field of surface plasmon polaritons travelling at the interface between the metal electrode and the organic layers. Quantitative calculations, treating the emitting molecules as classical electrical dipoles ( $c f$. the sketch on the title page of this article), reveal that in planar OLED stacks typically around $50 \%$ of the light is trapped in waveguided and plasmon modes [7]. As an example, Fig. 3 shows how the contribution of different optical channels varies as a function of the distance of the emission layer to the cathode, due to interference effects and coupling to trapped modes. It is therefore not surprising that developing new concepts for improving light extraction efficiency has been a major issue over recent years (for an overview see, e.g. Ref. [8]).

\section{Improving efficiency}

In the following some of these approaches will be highlighted. The extraction of light trapped in the glass substrate is quite straightforward. This fraction can be made accessible by modifications of the backside of the substrate, e.g., by micro-lens arrays or scattering foils, which are commercially available. Nevertheless, if the unique form factor of OLEDs (large-area, thin and flat) is to be preserved, these devices typically extract only part of the trapped light. Next, the propagation of waveguide modes can be suppressed by scattering at photonic crystal structures or by random scattering structures. The effectiveness of this approach relies on the spatial overlap of the waveguide modes with such features; in other words, they have to be employed close to the emission zone of the OLED [9]. Another approach relies on matching the refractive index of the organic materials, where the light is generated, with the substrate supporting the OLED layer stack [10]. Though impressive efficiency values larger than $40 \%$ have been reported using high-index glass substrates, the overall cost of the OLEDs would increase considerably by this approach. Finally, there is the contribution of surface plasmon losses. Owing to their evanescent nature, the simplest way to avoid them is to increase the distance between the emitter and the metal electrode. This means, however, that one has to increase the overall organic layer thickness, which in turn implies that more energy is coupled into waveguided modes. Scattering approaches, as discussed above in the context of waveguided modes, are also applicable to surface plasmons
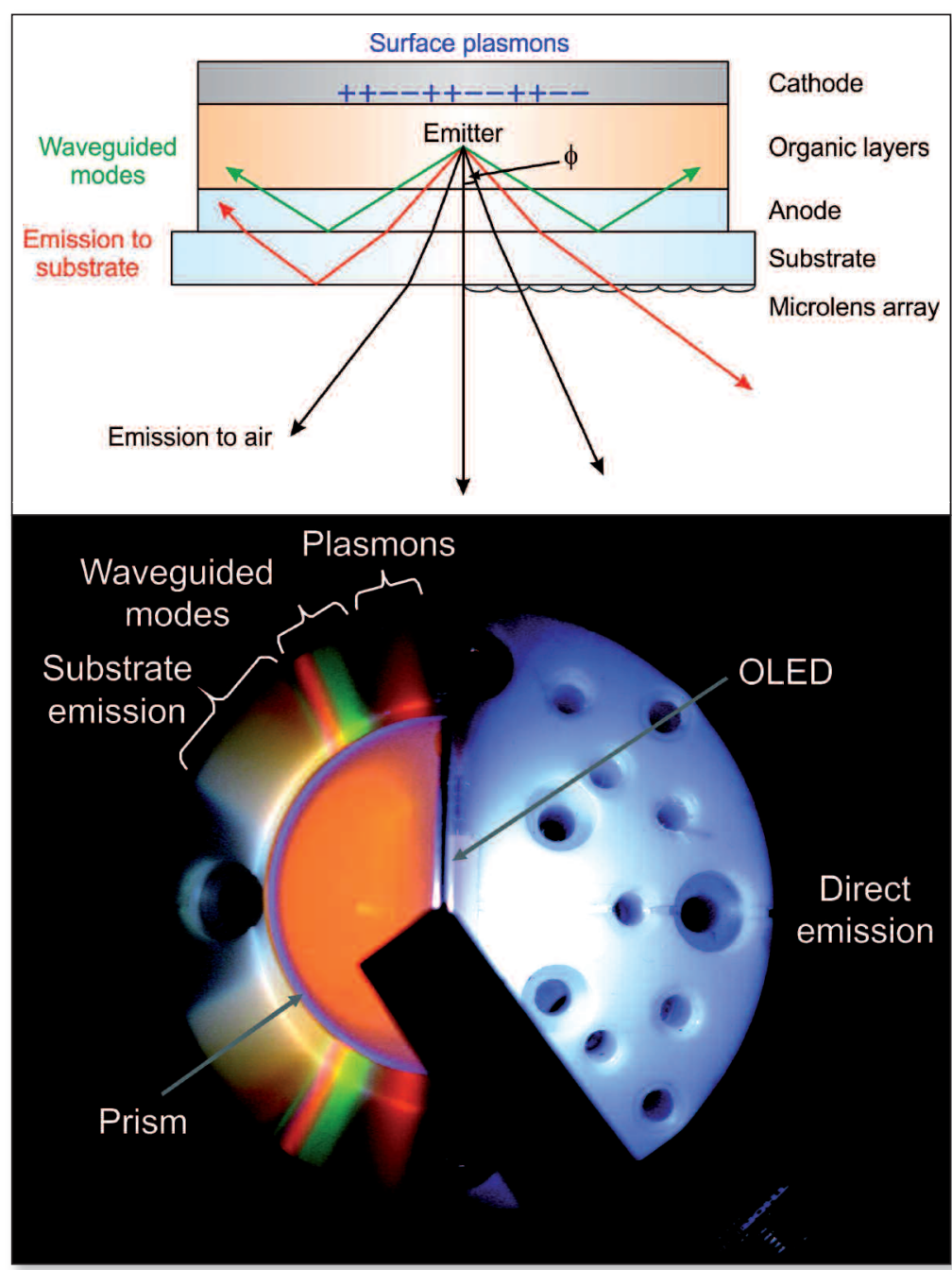

$\Delta$ FIG. 2: (a) Different optical modes to which an excited emitter molecule can dissipate its energy (see text for further details). Note that in a real OLED the substrate is much thicker than all the other layers. In (b) a high index prism is used in order to extract trapped light in a white OLED. The OLED is mounted vertically on a teflon table with the prism attached to its semitransparent metal cathode. 0 n the right hand side one can see the ordinary white light emission through the glass substrate, while on the left hand side different contributions are distinguishable under different viewing angles.

provided that the mode has sufficient overlap with the scatterers $[11,12]$. Another way to avoid the excitation of surface plasmons, even if the emitter is rather close to the metal, is to control the orientation of the emitting molecules and thus of their transition dipole moments (cf. the sketch on the title page). Keeping the radiation pattern of a classical electrical dipole in mind and considering that surface

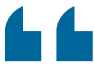

\section{Impressive numbers are obtained with white OLEDs reaching more than $60 \mathrm{Im} / \mathrm{W}$ ?}

transverse magnetic modes, one readily concludes that perfectly horizontally oriented dipoles would only very weakly couple to these plasmons. This effect has been known for many years in polymeric OLEDs. Only very recently, however, we have been able to show that 
orientation effects also play a role in small molecule OLEDs fabricated by vacuum deposition, where the fluorescent or phosphorescent dyes are embedded with only a few percent content in a matrix material [13].

\section{Conclusion}

By now, commercially available white OLEDs are specified with luminous efficacies of about $25 \mathrm{~lm} / \mathrm{W}$ [2], i.e., they are no more efficient than the best halogen lamps. However, several laboratories have reported values exceeding $60 \mathrm{~lm} / \mathrm{W}$ for devices with good colour compliance being currently under development. Of course there is room for improvement regarding the impressive numbers obtained with inorganic LEDs reaching more than $100 \mathrm{~lm} / \mathrm{W}$. But as already mentioned such a comparison is of limited relevance. A strong benefit of

V FIG. 3: (a) The amount of energy dissipated into different optical channels as a function of wavelength and wave-vector for a prototypical green OLED. Region (1) is direct emission, (2) stands for emission to the glass substrate, (3) represents waveguided modes and (4) surface plasmons. In (b) the relative contribution of different modes is plotted vs. the distance of the emission zone to the top electrode. (For details of the simulations we refer to Ref. [7].)
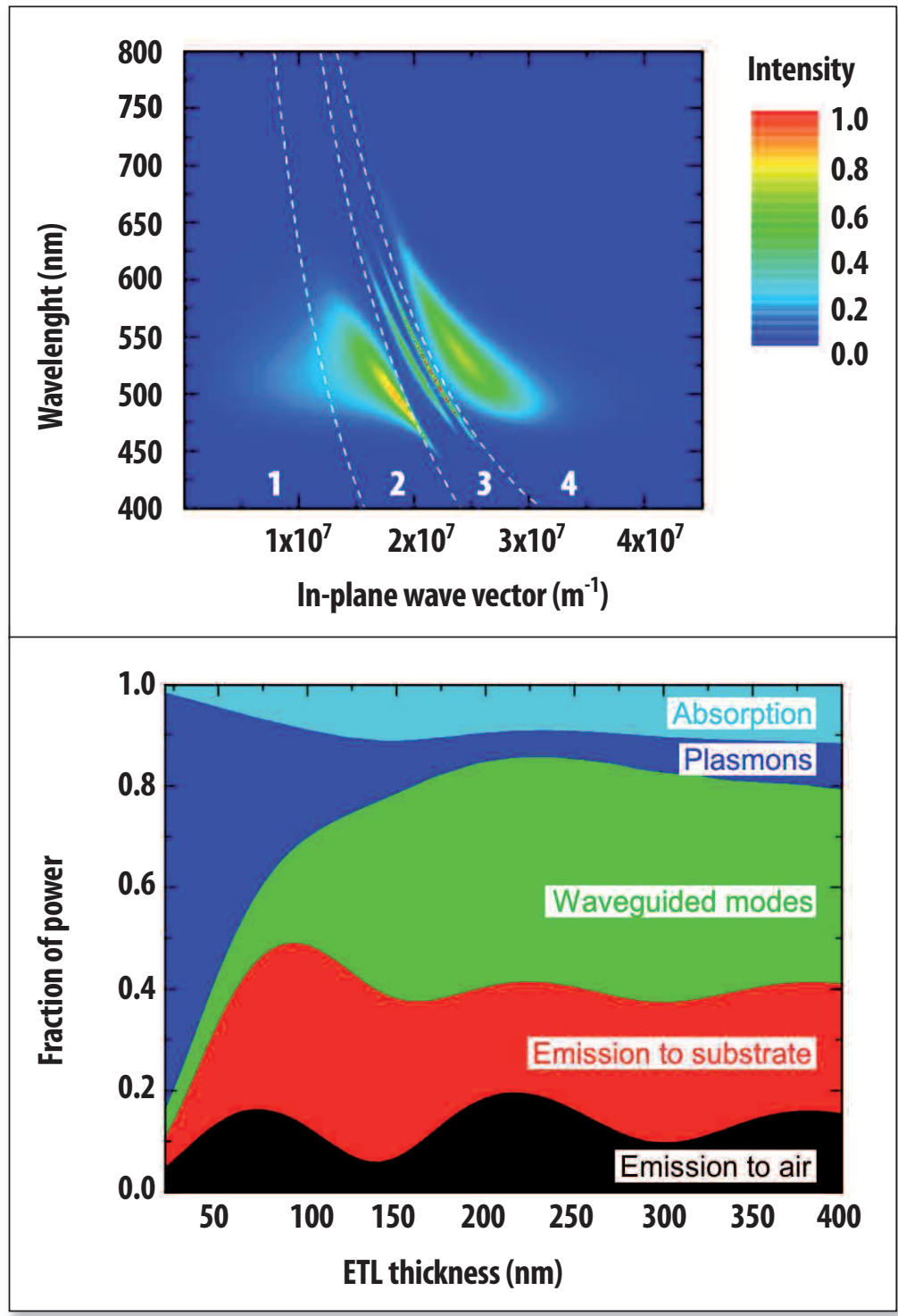

OLEDs is their unique form factor, with the light being distributed homogeneously over large area and thus being glare-free. Hence, (almost) no additional fixtures are needed: the OLED already is the luminaire!

\section{Acknowledgment}

The authors thank the German Ministry of Education and Research (BMBF) for funding within the TOPAS project (FKZ 13N10474) and colleagues from Osram Opto Semiconductors (Regensburg, Germany) and Fraunhofer Institute for Applied Optics and Precision Engineering (Jena, Germany) for continuous support and fruitful discussions.

\section{About the authors}

Jörg Frischeisen, Bert Scholz and Tobias Schmidt are $\mathrm{PhD}$ students at the Institute of Physics at Augsburg University. Wolfgang Brütting is professor in experimental solid state physics and leads the research group on organic semiconductors at Augsburg University.

\section{References}

[1] C.W. Tang, S.A. VanSlyke, Appl. Phys. Lett. 51 (1987) 913; J.H. Burroughes, D.D.C. Bradley, A.R. Brown, R.N. Marks, K. Mackay, R.H. Friend, P.L. Burns, A.B. Holmes, Nature 347 (1990) 539.

[2] Since 2010 white OLED panels are commercially available, e.g. from Osram (www.osram.com/osram com/LED/OLED Lighting/index.html) and Philips (www.lighting.philips.com/ main/lightcommunity/trends/oled/index.wpd).

[3] Y.-S. Tyan, J. Photonics for Energy 1 (2011) 011009; M. Gather A. Köhnen, K. Meerholz, Adv. Mater. 23 (2011) 233.

[4] J. Kido, K. Okuyama, K. Nagai, Appl. Phys. Lett. 64 (1994) 815.

[5] M.A. Baldo, D.F. O'Brien, Y. You, A. Shoustikov, S. Sibley, M.E. Thompson, S.R. Forrest, Nature 395 (1998) 151

[6] S. Reineke, F. Lindner, G. Schwartz, N. Seidler, K. Walzer, B. Lüssem, K. Leo, Nature 459 (2009) 234.

[7] S. Nowy, B.C. Krummacher, J. Frischeisen, N.A. Reinke, W. Brütting, J. Appl. Phys. 104 (2008) 123109.

[8] K. Saxena, V.K. Jain, D.S. Mehta, Optical Materials 32 (2009) 221.

[9] J.M. Lupton, B.J. Matterson, I.D.W. Samuel, M.J. Jory, W.L. Barnes, Appl. Phys. Lett. 77 (2000) 3340.

[10] G. Gaertner, H. Greiner, Proc. SPIE 6999 (2008) 69992T; S. Mladenovski, K. Neyts, D. Pavicic, A. Werner, C. Rothe, Opt. Express 17 (2009) 7562.

[11] S. Wedge, A. Gianattasio, W.L. Barnes, Organic Electronics 8 (2007) 136.

[12] J. Frischeisen, Q. Niu, A. Abdellah, J.B. Kinzel, R. Gehlhaar G. Scarpa, C. Adachi, P. Lugli, W. Brütting, Opt. Express 19 (2011) A7.

[13] J. Frischeisen, D. Yokoyama, A. Endo, C. Adachi, W. Brütting Organic Electronics 12 (2011) 809; M. Flämmich, J. Frischeisen, D.S. Setz, D. Michaelis, B.C. Krummacher, T.D. Schmidt, W. Brütting, N. Danz, Organic Electronics (2011) in press.

[14] T. Tsutsui, E. Aminaka, Philos. Trans. R. Soc. London, Ser. A 355 (1997) 801. 